Liliane Cupertino de Assis ${ }^{1}$

(D) https://orcid.org/0000-0002-4937-7189

Camyla Rocha de Carvalho

Guedine ${ }^{2}$

Ohttps://orid.org/0000-0002-7026-4314

Pedro Henrique Berbert de

Carvalho ${ }^{3}$

OChttps://orcid.org/0000-0002-4918-5080

\section{Uso da mídia social e sua associação com comportamentos alimentares disfuncionais em estudantes de Nutrição}

\author{
Social media use and its association with disordered \\ eating among Nutrition Science students
}

DOl: $10.1590 / 0047-2085000000288$

\section{RESUMO}

Objetivos: Avaliar a associação entre o uso da mídia social e comportamentos alimentares disfuncionais e identificar preditores desses comportamentos. Métodos: Estudo realizado com 207 estudantes de Nutrição. Foram aplicadas três medidas para obter dados sociodemográficos, uso da mídia social e comportamentos alimentares disfuncionais (Eating Attitudes Test-26). A associação entre o uso da mídia social e os comportamentos alimentares disfuncionais foi verificada pelo teste qui-quadrado de Pearson $(p<0,05)$. Regressão logística binária foi conduzida para avaliar preditores dos comportamentos alimentares disfuncionais, considerando as razões de chance. Resultados: Comportamentos alimentares disfuncionais foram verificados em $27,9 \%$ dos estudantes de Nutrição. As variáveis de uso da mídia social associadas aos comportamentos alimentares disfuncionais incluem o hábito de acompanhar dicas alimentares e de consumir alimentos sugeridos pela mídia, o tempo de uso, seguir dieta e/ou orientação nutricional, motivação para seguir uma dieta sem um acompanhamento nutricional e consumo de alimentos e fármacos para a perda de peso. Os preditores dos comportamentos alimentares disfuncionais foram: "Já consumi alimentos propostos pela mídia com o intuito de perda de peso" e "Ao observar corpos disseminados como 'belos' pela mídia, me sinto motivado a seguir uma dieta sem um acompanhamento nutricional adequado por um nutricionista". Conclusões: Diversas crenças e comportamentos orientados ao uso da mídia social estão associados aos comportamentos alimentares disfuncionais em estudantes de Nutrição, em que se destacam maiores razões de chances para o desenvolvimento de comportamentos de risco quando há consumo de alimentos para perda de peso e motivação para seguir dieta sem acompanhamento nutricional, mesmo por esse grupo.

\section{PALAVRAS-CHAVE}

Comportamento alimentar, mídia social, transtornos da alimentação e da ingestão de alimentos, saúde do estudante.

\section{ABSTRACT}

Objectives: To assess the association between the use of social media and disordered eating and to identify predictors of disordered eating. Methods: Study carried out with 207 Nutrition Science students. Three measures were applied to evaluate sociodemographic data, the use of social media, and disordered eating (Eating Attitudes Test-26). Associations between the use of social media and disordered eating were evaluated by the Chi-square test ( $p<0.05)$. Binary logistic regression was conducted to assess predictors of disordered eating, considering the odds ratios. Results: Disordered eating was observed in $27.9 \%$ of Nutrition Science students. Variables of the use of social media associated with disordered eating include following dietary tips and consuming foods suggested by the media, the time of social media use, dieting or following nutritional guidance, motivation to engage in a diet without nutritional monitoring, consumption of food and drugs for weight loss. Predictors of disordered eating were "I have consumed food proposed by the media, with the aim of losing weight" and "When observing 'beauty' bodies disseminated by the media, I feel motivated to follow a diet without a nutritional monitoring of a nutritionist". Conclusions: Several beliefs and behaviors oriented to the use of social media were associated with disordered eating in Nutrition Science students, in which there were greater odds ratio of developing risky behaviors when consuming food for weight loss and motivation to follow a diet without nutritional monitoring, even in Nutrition Science students.

\section{KEYWORDS}

Feeding behavior, social media, feeding and eating disorders, student health.

Received in: Apr/15/2020. Approved in: July/21/2020

1 Universidade Federal de Juiz de Fora (UFJF), Curso de Bacharelado em Nutrição, Governador Valadares, MG, Brasil.

2 Universidade Federal do Acre (UFAC), Centro de Ciências da Saúde e do Desporto, Curso de Bacharelado em Nutrição, Rio Branco, AC, Brasil.

3 Universidade Federal de Juiz de Fora (UFJF), Instituto de Ciências da Vida, Departamento de Educação Física, Governador Valadares, MG, Brasil.

Address for correspondence: Pedro de Carvalho. Rua das Garças, 121, ap. 201, Lagoa Santa Governador Valadares - 35065-110 - Minas Gerais, MG, Brasil. E-mail: pedro.berbert@uff.edu.br 


\section{INTRODUÇÃO}

Conceituar o comportamento alimentar é algo complexo, pois o ato de comer vai além das necessidades básicas da alimentação'. O conceito abarca práticas alimentares em associação a atributos culturais, sociais e religiosos, e as formas de convívio com o alimento, ou seja, as práticas alimentares, não se resumem apenas aos alimentos que são ingeridos, mas também às escolhas inseridas em cada indivíduo através de gerações e às sensações proporcionadas pelos sentidos ${ }^{1,2}$.

A transição da adolescência para a idade adulta jovem é um estágio único em que a maioria das pessoas passa por mudanças no comportamento alimentar ${ }^{3-5}$. O ingresso no ensino superior, por exemplo, pode ocasionar a mudança de uma vida de dependência como adolescente para uma vida de completa independência alimentar ${ }^{3,4}$, de modo que, não raramente, jovens universitários desenvolvem comportamentos alimentares disfuncionais ${ }^{6}$. Estes podem ser entendidos como comportamentos alimentares problemáticos, tais como práticas purgativas, compulsão e/ou restrição alimentar, além de outros métodos inadequados para perder ou controlar o peso, que ocorrem com menor frequência ou são menos graves do que aqueles necessários para atender a todos os critérios diagnósticos de um transtorno alimentar ${ }^{7}$.

Dentre os fatores de influência sobre o comportamento alimentar de jovens adultos, destaca-se o uso da mídia social $\left.\right|^{3,48-10}$ e sua consequente influência sobre as causas relacionadas aos comportamentos alimentares disfuncionais, como a insatisfação corporal e a internalização dos ideais de corpo. O modelo teórico biopsicossocial ${ }^{10}$ propõe que o uso da mídia social é determinante na internalização de ideais de corpo propagados socialmente, que, por sua vez, pode gerar dois desfechos: a insatisfação corporal e a restrição alimentar. Cabe destacar que esses últimos são considerados fatores de risco para o desenvolvimento de transtornos alimentares, sendo considerados ainda preditores dos comportamentos alimentares disfuncionais ${ }^{11}$. Portanto, entender a internalização desses ideais é fundamental.

A internalização é um processo cognitivo complexo e diz respeito às crenças e aos comportamentos adotados pelos sujeitos ${ }^{12}$. Uma crença comum sugere que a aquisição de um corpo "magro" por parte das mulheres traria um sentimento de felicidade e elevado status social'13.

Profissionais de saúde e estudantes, enquanto seres sociais, não estão imunes às influências socioculturais ${ }^{14}$. Estudos apontam que, entre as diversas áreas de formação em saúde, estudantes de Educação Física e de Nutrição são aqueles que possuem as mais altas taxas de prevalência de comportamentos alimentares disfuncionais e insatisfação corporal $^{5,15}$. Em se tratando de graduandos de Nutrição, aumenta a importância de novos estudos, pois, se profissionais do campo da saúde e da Nutrição, apesar de sua expertise nesses domínios, desejam atender a essas normas, evidencia-se a importância do tema como questão sociossanitária'16,17.

Compreender, portanto, a associação entre o uso da mídia social e o comportamento alimentar de estudantes de Nutrição pode auxiliar no desenvolvimento de novas estratégias contra a internalização de algumas crenças disfuncionais e atitudes inadequadas em relação ao corpo e à comida, já que a expectativa é de que, enquanto futuros profissionais, eles sejam cuidadores saudáveis, capazes de cuidar de outros sujeitos ${ }^{16-18}$. Até o presente momento, não é de nosso conhecimento nenhum estudo que tenha investigado em profundidade aspectos cognitivos (crenças) e atitudinais (sentimentos e comportamentos) em relação ao uso da mídia social e sua associação com comportamentos alimentares disfuncionais em estudantes de Nutrição.

As produções existentes no campo dos comportamentos alimentares disfuncionais em jovens universitários têm se direcionado especialmente à avaliação de sua prevalência 17,19,20, à comparação de estudantes de diferentes áreas da saúde ${ }^{21,22}$ e à investigação de fatores associados, como, por exemplo, os distúrbios de imagem corporal5,21,23. Especificamente sobre o uso da mídia social e sua associação com comportamentos alimentares disfuncionais, os estudos têm se dedicado à análise do tempo/frequência de uso ${ }^{24-26}$, às plataformas/aplicativos mais utilizados ${ }^{25,27}$ e à realização de estudos experimentais com exposição à mídia25,26.

Desse modo, o presente estudo teve como objetivo principal avaliar a associação entre o uso da mídia social e comportamentos alimentares disfuncionais de estudantes do curso de Nutrição, e como objetivo secundário, verificar os preditores de comportamentos alimentares disfuncionais em estudantes de Nutrição.

\section{MÉTODOS}

\section{Amostra}

Este estudo do tipo transversal foi realizado com estudantes de ambos os sexos, maiores de 18 anos, matriculados em um curso superior de Nutrição, do estado de Minas Gerais, Brasil. Foram considerados critérios de inclusão a participação voluntária e a presença em sala de aula no momento de coleta de dados. Foram excluídos aqueles que, por algum motivo, deixaram de responder a mais de 5\% das perguntas (protocolo de pesquisa) ou que autorrelataram algum transtorno mental diagnosticado previamente (depressão, transtorno alimentar, entre outros).

Para obter uma amostra representativa de estudantes de Nutrição, foi realizado cálculo amostral para amostras finitas, considerando o número de estudantes de Nutrição matriculados na instituição de ensino pesquisada no momento da realização do presente estudo (249 alunos matriculados). 
Consideraram-se, ainda, a prevalência média de 30\% de comportamentos alimentares disfuncionais em estudantes de Nutrição ${ }^{17,19,28}$, o grau de confiança de 95\% e a margem de erro de 5\%. Com esses parâmetros, determinou-se um mínimo de 152 participantes na presente investigação.

\section{Aspectos éticos}

A presente pesquisa está de acordo com os princípios da Declaração de Helsinki e da Resolução no 466/2012 do Conselho Nacional de Saúde do Brasil. Foi aprovada pelo Comitê de Ética e Pesquisa em Seres Humanos da instituição de ensino superior na qual os dados foram coletados, com a inscrição n 02613618.0.0000.5147 e o parecer de aprovação no 3.106.674. Todos os participantes concordaram em participar da pesquisa e assinaram o Termo de Consentimento Livre e Esclarecido.

\section{Instrumentos}

\section{Dados sociodemográficos}

Para a obtenção das características gerais dos estudantes, foi estruturado um questionário específico para esta pesquisa com as seguintes variáveis: sexo, idade, massa corporal e estatura autorreferidas (para o cálculo do índice de massa corporal - IMC ${ }^{29}$ ) e ano de ingresso ao curso. Ainda nesse questionário foi avaliado o nível socioeconômico por meio do Critério de Classificação Econômica Brasil ${ }^{30}$. Após o somatório dos itens do instrumento, os indivíduos foram classificados nos seguintes cortes: A, B1, B2, C1, C2, D-E $E^{30}$.

Para avaliar o estado nutricional antropométrico dos estudantes, foi utilizado o IMC, obtido por meio da relação entre o peso $(\mathrm{kg})$ e o quadrado da altura $\left(\mathrm{m}^{2}\right)$, cujos dados foram autorreferidos. Os participantes foram classificados em baixo peso (menor que 18,5 kg/m²), peso normal $\left(18,5-24,9 \mathrm{~kg} / \mathrm{m}^{2}\right.$ ), sobrepeso $\left(25,0-29,9 \mathrm{~kg} / \mathrm{m}^{2}\right)$ e obesidade $\left(30,0-40,0 \mathrm{~kg} / \mathrm{m}^{2}\right)^{29}$.

\section{Uso da mídia social}

Para avaliar crenças, sentimentos e comportamentos em relação ao corpo e à comida quando do uso da mídia social, foi desenvolvido um conjunto de 10 perguntas/afirmativas (ver tabela 1), respondidas em uma escala do tipo Likert de cinco pontos ( 1 = concordo totalmente a $5=$ discordo totalmente). As questões centrais envolviam a análise do tempo de uso da mídia social, hábitos alimentares, prática de dietas provindas da mídia, realização de dietas sem acompanhamento nutricional, uso de fármacos, suplementos e anabolizantes, entre outros. Tais questões foram formuladas com base em estudos prévios sobre o tema ${ }^{24-27}$.

O tempo de uso de mídia social foi dividido em três grupos, a saber: tempo de exposição "menor que 30 minutos", "entre 30 e 60 minutos" e "maior que 60 minutos". Para as demais perguntas, os resultados foram agrupados de modo que os participantes que se enquadravam entre as variáveis concordo totalmente (1) e concordo parcialmente (2) foram considerados como "concordo" e os que se enquadravam entre as variáveis não concordo e nem discordo (3), discordo parcialmente (4) e discordo totalmente (5) foram considerados como "discordo".

\section{Comportamentos alimentares disfuncionais}

Para avaliar comportamentos alimentares disfuncionais, foi utilizado o Eating Attitudes Test-26 (EAT-26) ${ }^{31,32}$. O EAT-26 avalia comportamentos alimentares restritivos - como dieta e jejum - e bulímicos - como ingestão excessiva de alimentos e vômitos provocados. $O$ instrumento possui 26 itens que devem ser respondidos em uma escala do tipo Likert de pontos, variando de 0 a 3 pontos. $O$ escore total é obtido pela soma das pontuações, podendo variar de 0 a 78, e quanto maior o escore obtido, maiores os comportamentos alimentares disfuncionais ${ }^{31}$. De acordo com a padronização dos escores do EAT-26, os participantes que obtêm pontuação maior ou igual a 21 são considerados de risco para o desenvolvimento de transtornos alimentares e aqueles com pontuação igual ou abaixo de 20 são considerados isentos de risco ${ }^{31}$.

\section{Procedimentos}

Após a aprovação pelo Comitê de Ética em Pesquisa da instituição de ensino, por meio da coordenação do curso, foi obtida a listagem de todos os alunos matriculados no curso de Nutrição no semestre de desenvolvimento da pesquisa. Aqueles regularmente matriculados e frequentes no curso foram convidados a participar do estudo. Houve a realização de uma reunião com o chefe do Departamento de Nutrição, solicitando apoio da coordenação e colaboração dos docentes na coleta de dados deste projeto.

O questionário foi aplicado durante os meses de agosto a outubro de 2019, sendo respondido individualmente pelos alunos do curso de Nutrição, após uma breve explicação sobre o trabalho. Sua aplicação foi feita a partir de um agendamento com docentes do curso de Nutrição. Os questionários foram respondidos em sala de aula, no início delas e com a presença dos docentes do curso. Foi esclarecido aos alunos que a participação era voluntária, sem prejuízo na disciplina em questão ou em qualquer setor do curso. Além disso, não foi estipulado tempo máximo para responder ao protocolo de pesquisa.

\section{Análise estatística}

Foi realizada estatística descritiva dos dados categóricos por meio de frequência absoluta e relativa. Média e desvios-padrão foram utilizados para descrever dados contínuos. Para verificar a associação entre o uso da mídia social e os comportamentos alimentares disfuncionais, foram conduzidas análises por meio do teste qui-quadrado de Pearson. 
A fim de verificar os melhores preditores dos comportamentos alimentares disfuncionais em estudantes de Nutrição, foi realizada regressão logística binária, utilizando no modelo preditivo todas as variáveis de uso de mídia social que demonstraram associação significante com os comportamentos alimentares disfuncionais. Para tanto, foram previamente avaliados os pressupostos de ausência de multicolinearidade (variance inflation factor menor do que 5) e outliers.

Uma série de análises de regressão logística binária foi realizada, incluindo (regressão stepwise forward) e retirando (regressão stepwise backward) variáveis no modelo até que se atingisse aquele como melhor ajustamento aos dados. 0 julgamento do modelo mais ajustado se deu pela análise de uma série de indicadores, a saber: teste qui-quadrado, teste de Hosmer-Lemershow, $\mathrm{R}^{2}$ de Nagelkerk e odds ratio (OR), considerando os intervalos de confiança (IC95\% OR).

A fim de explorar indicadores de validade e confiabilidade da escala desenvolvida para avaliação do uso da mídia social, foi realizada uma análise fatorial exploratória, seguida de análise de confiabilidade por meio do coeficiente ômega (McDonald's $\omega$ ). O índice Kaiser-Mayer-Olkin (igual a 0,76 ) e o teste de esfericidade de Bartlett $\left(x^{2}(36)=352,313, p<0,001\right)$ indicaram adequação dos dados para fatoração. A análise fatorial exploratória por fatoração do eixo principal, utilizando o critério Kaiser, a análise paralela e a inspeção do gráfico de declividade para a extração de fatores indicaram a presença de fator único. Todos os itens da escala apresentaram carga fatorial superior a 0,40. A consistência interna da escala foi adequada $(\omega=0,73)$.

Os dados foram tratados no software SPSS v. 21.0, adotando nível de significância de 5\%. Especificamente para a análise fatorial exploratória e a análise da consistência interna da escala de uso da mídia social, recorreu-se ao software JASP v. 0.12.1.

\section{RESULTADOS}

O presente estudo foi realizado com 207 participantes, havendo uma perda amostral de seis participantes, devido às irregularidades no preenchimento dos questionários. Nenhum participante relatou possuir diagnóstico de algum transtorno mental. Desse modo, foram analisados dados de 201 estudantes de Nutrição (84,6\% do sexo feminino; $n=170$ ).

A média de idade dos participantes foi de 21,73 (DP $=4,4$ anos). Quanto à cor/raça, os participantes se autodeclararam majoritariamente como pardos $(n=91 ; 45,3 \%)$ e brancos $(n=87 ; 43,3 \%)$, seguidos de negros $(n=23 ; 11,4 \%)$. Em relação ao momento do curso de Nutrição, 32,3\% ( $n=65)$ estavam no primeiro ano do curso, $20,4 \%(n=41)$, no segundo, $22,8 \%(n=46)$, no terceiro, $14,5 \%(n=29)$, no quarto e $10 \%$ $(n=20)$, no quinto ano.
Em relação à massa corporal, os participantes apresentavam as seguintes classificações: baixo peso $(n=18 ; 8,9 \%)$, peso normal $(n=130 ; 64,7 \%)$, sobrepeso $(n=45 ; 22,4 \%)$ e obesidade ( $n=8 ; 4 \%$ ). Quanto ao nível socioeconômico, observou-se que a grande maioria dos participantes era das classes B2 ( $n=63,31,3 \%)$ e C1 ( $n=55,27,4 \%)$, seguidas das classes C2 ( $n=32,15,9 \%)$ e B1 ( $n=31,15,4 \%)$, enquanto a minoria foi representada pelas classes $A(n=12 ; 6 \%)$ e D-E $(n=8 ; 4 \%)$.

No tocante aos comportamentos alimentares disfuncionais, observou-se que 27,9\% $(n=56)$ apresentaram escores iguais ou superiores a 21 no EAT-26, indicando risco para o desenvolvimento de transtornos alimentares (EAT+).

Dentre os 201 participantes, 52,74\% ( $n=106)$ têm o hábito de acompanhar dicas alimentares na mídia, 20,4\% ( $n=$ 41) têm o hábito de se alimentar com base em conteúdos disseminados pela mídia, enquanto $30,85 \%(n=62)$ já seguiram dieta e/ou orientação nutricional provinda da mídia. Do total, $96,52 \%(n=194)$ acreditam que a mídia influencia o comportamento alimentar das pessoas, e 35,82\% ( $n=72)$ já consumiram alimentos propostos pela mídia com o intuito de perda de peso, 11,94\% ( $n=24)$ já fizeram uso de fárma$\cos , 17,91 \%(n=36)$ já usaram suplementos e 1,49\% ( $n=3)$ já usaram esteroides anabolizantes por sugestão de algum canal de mídia. Por fim, 32,34\% $(n=65)$ relataram que, ao observar corpos disseminados como "belos" pela mídia, se sentiram motivados a seguir uma dieta sem um acompanhamento nutricional adequado por um nutricionista.

A tabela 1 apresenta os dados descritivos de uso de mídia social e sua associação com os comportamentos alimentares disfuncionais dos estudantes de Nutrição. Com exceção da crença de que a mídia influencia o comportamento alimentar das pessoas e o comportamento de uso de suplementos e esteroides anabólicos por sugestão de algum canal de mídia, todos os demais itens da escala de uso da mídia social demonstraram associação com os comportamentos alimentares disfuncionais (Tabela 1).

A análise de regressão logística binária demonstrou que os preditores dos comportamentos alimentares disfuncionais em estudantes de Nutrição foram "Já consumi alimentos propostos pela mídia com o intuito de perda de peso" e "Ao observar corpos disseminados como 'belos' pela mídia, me sinto motivado a seguir uma dieta sem um acompanhamento nutricional adequado por um nutricionista" $\left(X^{2}(2)=54,645 ; p<0,0001, R^{2}\right.$ Negelkerk $\left.=0,343\right)$. O teste de Hosmer-Lemershow demonstrou que as categorias previstas são iguais às categorias esperadas $\left(X^{2}(2)=0,85 ; p<0,959\right)$. As demais questões referentes ao uso da mídia não acrescentaram explicação ao modelo e, portanto, foram retiradas do modelo final. O modelo de regressão logística binária mais ajustado pode ser verificado na tabela 2. Destaca-se que os pressupostos de ausência de outliers e multicolinearidade foram atendidos. 
Tabela 1. Análise descritiva do uso de mídia social e sua associação com comportamentos alimentares disfuncionais $(\mathrm{n}=201)$

\begin{tabular}{|c|c|c|c|c|}
\hline & EAT- & EAT+ & $\chi^{2}(\mathbf{g l})$ & p-valor \\
\hline & n (\%) & n (\%) & & \\
\hline \multicolumn{5}{|c|}{ Tenho o hábito de acompanhar dicas alimentares na mídia. } \\
\hline Concordo & $68(46,9 \%)$ & $38(67,9 \%)$ & \multirow[t]{2}{*}{$\chi^{2}(1)=7,121$} & $0,008^{*}$ \\
\hline Discordo & $77(53,1 \%)$ & $18(32,1 \%)$ & & \\
\hline \multicolumn{5}{|c|}{ Quanto tempo por dia você costuma gastar acompanhando dicas alimentares na mídia? } \\
\hline Menos de 30 & $81(55,9 \%)$ & $22(39,3 \%)$ & \multirow[t]{3}{*}{$\chi^{2}(2)=8,676$} & \multirow[t]{3}{*}{$0,013^{*}$} \\
\hline $30-60$ & $43(29,7 \%)$ & $16(28,6 \%)$ & & \\
\hline Mais de 60 & $21(14,5 \%)$ & $18(32,1 \%)$ & & \\
\hline \multicolumn{5}{|c|}{ Tenho hábito de me alimentar com base em conteúdos disseminados pela mídia. } \\
\hline Concordo & $23(15,9 \%)$ & $18(32,1 \%)$ & \multirow[t]{2}{*}{$\chi^{2}(1)=6,595$} & \multirow[t]{2}{*}{$0,01^{*}$} \\
\hline Discordo & $122(84,1 \%)$ & $38(67,9 \%)$ & & \\
\hline \multicolumn{5}{|c|}{ Já segui dieta e/ou orientação nutricional provinda da mídia. } \\
\hline Concordo & $35(24,1 \%)$ & $27(48,2 \%)$ & \multirow[t]{2}{*}{$\chi^{2}(1)=10,978$} & \multirow[t]{2}{*}{$0,001^{*}$} \\
\hline Discordo & $110(75,9 \%)$ & $29(51,8 \%)$ & & \\
\hline \multicolumn{5}{|c|}{ Acredito que a mídia influencia o comportamento alimentar das pessoas. } \\
\hline Concordo & $138(95,2 \%)$ & $56(100 \%)$ & $\chi^{2}(1)=2,801^{\#}$ & 0,094 \\
\hline Discordo & $7(4,8 \%)$ & $0(0,0 \%)$ & & \\
\hline \multicolumn{5}{|c|}{$\begin{array}{l}\text { Ao observar corpos disseminados como "belos" pela mídia, me sinto motivado a seguir uma } \\
\text { dieta sem um acompanhamento nutricional adequado por um nutricionista. }\end{array}$} \\
\hline Concordo & $32(22,1 \%)$ & $33(58,9 \%)$ & \multirow[t]{2}{*}{$\chi^{2}(1)=25,084$} & \multirow[t]{2}{*}{$0,0001^{*}$} \\
\hline Discordo & $113(77,9 \%)$ & $23(41,1 \%)$ & & \\
\hline \multicolumn{5}{|c|}{ Já consumi alimentos propostos pela mídia com o intuito de perda de peso. } \\
\hline Concordo & $31(21,4 \%)$ & $41(73,2 \%)$ & \multirow[t]{2}{*}{$\chi^{2}(1)=47,215$} & \multirow[t]{2}{*}{$0,0001^{*}$} \\
\hline Discordo & $114(78,6 \%)$ & $15(26,8 \%)$ & & \\
\hline \multicolumn{5}{|c|}{ Já fiz uso de fármacos por sugestão de algum canal de mídia. } \\
\hline Concordo & $11(7,6 \%)$ & $13(23,2 \%)$ & \multirow[t]{2}{*}{$\chi^{2}(1)=9,384$} & \multirow[t]{2}{*}{$0,002^{*}$} \\
\hline Discordo & $134(92,4 \%)$ & $43(76,8 \%)$ & & \\
\hline \multicolumn{5}{|c|}{ Já fiz uso de suplementos por sugestão de algum canal de mídia. } \\
\hline Concordo & $23(15,9 \%)$ & $13(23,2 \%)$ & $\chi^{2}(1)=1,485$ & 0,223 \\
\hline Discordo & $122(84,1 \%)$ & $43(76,8 \%)$ & & \\
\hline \multicolumn{5}{|c|}{ Já fiz uso de esteroides anabolizantes por sugestão de algum canal de mídia. } \\
\hline Concordo & $2(1,4 \%)$ & $1(1,8 \%)$ & \multirow[t]{2}{*}{$\chi^{2}(1)=0,045^{\#}$} & \multirow[t]{2}{*}{1,000} \\
\hline Discordo & $143(98,6 \%)$ & $55(98,2 \%)$ & & \\
\hline
\end{tabular}

EAT-: grupo sem comer transtornado (escore < 21 pontos no EAT-26); EAT+: grupo com comer transtornado (escore $\geq 21$ pontos no EAT-26); $\chi^{2}$ (gl): teste qui-quadrado e grau de liberdade; $n$ (\%): frequência absoluta e relativa.

${ }^{*} p<0,05$.

\# Utilizado teste exato de Fisher.

Tabela 2. Análise de regressão logística binária para identificação de preditores dos comportamentos alimentares disfuncionais em estudantes de Nutrição $(n=201)$

\begin{tabular}{lcccccc}
\hline Variáveis & B & SE & Wald & gl & p-valor & OR [IC95\%] \\
\hline Já consumi alimentos propostos pela mídia com o intuito de perda de peso. & 2,012 & 0,379 & 28,245 & 1 & $0,000^{*}$ & $7,478[3,561-15,704]$ \\
$\begin{array}{l}\text { Ao observar corpos disseminados como "belos" pela mídia, me sinto motivado a seguir } \\
\text { uma dieta sem um acompanhamento nutricional adequado por um nutricionista. }\end{array}$ & 1,074 & 0,378 & 8,061 & 1 & $0,005^{*}$ & $2,927[1,395-6,142]$ \\
\begin{tabular}{l} 
Constante \\
\hline
\end{tabular} & $-0,774$ & 0,305 & 6,444 & 1 & 0,011 & 0,461 \\
\hline
\end{tabular}

B: beta; SE: erro-padrão; gl: graus de liberdade; OR: odds ratio (razão de chances); IC95\%: intervalo de confiança de 95\%.

${ }^{*} p<0,05$. 


\section{DISCUSSÃO}

Em nossa pesquisa, verificou-se uma associação entre crenças, sentimentos e comportamentos relacionados ao uso da mídia social e comportamentos alimentares disfuncionais. Foi identificada prevalência de $27,9 \%$ de comportamentos alimentares disfuncionais nos estudantes de Nutrição, prevalência essa próxima àquela estimada em estudos similares ${ }^{17,19,28}$.

Destaca-se o fato de que a prevalência é maior em estudantes que utilizam a mídia social por maior tempo diário. Participantes que ficam menos de 30 minutos apresentam prevalência de comportamentos alimentares disfuncionais menor do que aqueles que ficam de 30 a 60 minutos. Além disso, aqueles que utilizam as redes sociais por mais de 60 minutos diários possuem o dobro de prevalência de comportamentos alimentares disfuncionais quando comparados àqueles que se conectam por 30 minutos ou menos. Resultados semelhantes foram observados em estudo realizado com jovens adultos ${ }^{8}$, no qual foi avaliada a associação entre o volume e a frequência de uso da mídia social e preocupações com a alimentação. Revisão sistemática da literatura ${ }^{25}$ aponta que essa associação é frequentemente encontrada nos estudos científicos até então realizados e destaca a necessidade de avaliação da associação entre o tempo de uso da mídia social e comportamentos alimentares disfuncionais em diversas populações. Nesse sentido, faz-se imperativo orientar os estudantes sobre a necessidade de limitar o tempo de uso da mídia social.

Ademais, é necessário estimular uma visão crítica sobre os conteúdos acessados no período de uso da mídia social. Isso porque nossos resultados apontam que aqueles que seguem dicas alimentares da mídia possuem maior prevalência de comportamentos alimentares disfuncionais. Esses possuem aproximadamente o dobro da prevalência de comportamentos alimentares disfuncionais em relação àqueles que não possuem esse comportamento. É interessante observar que quase a totalidade dos estudantes de Nutrição avaliada concorda que o uso de mídia social afeta o comportamento alimentar das pessoas e que, ainda assim, metade dos participantes refere ter o hábito de acompanhar dicas alimentares na mídia. Tal fato reforça a necessidade de aprofundar discussões sobre o comportamento alimentar e sua associação com o uso da mídia entre os estudantes.

Estudo realizado com universitários dos cursos de Psicologia e Nutrição $0^{33}$ identificou que aqueles que tinham por hábito realizar "dietas da moda" apresentavam maior prevalência de comportamentos alimentares disfuncionais. Apesar de os estudantes de Nutrição apresentarem maior conhecimento teórico, eles ainda seguem dietas da moda e serão futuros profissionais que lidarão diretamente com o corpo e com o público. Suas preocupações com o próprio corpo podem refletir na sua prática profissional e influenciar seus futuros alunos ou clientes ${ }^{16-18,22}$.
Vale destacar que, em nosso estudo, mais da metade dos estudantes de Nutrição relataram ter consumido alimentos propostos pela mídia com o intuito de perda de peso. Isso pode ser explicado pela diferença entre o peso atual dos indivíduos e o ideal promovido pela mídia, associado com a necessidade de adaptação à sociedade e uma baixa autoestima, fatores que podem determinar uma insatisfação corporal. E essa insatisfação pode levar muitas vezes ao início de comportamentos de risco para transtornos alimentares, como o uso de dietas restritivas e/ou dietas da moda, além de métodos compensatórios para controle de peso ${ }^{19}$.

Estudo realizado com estudantes universitários indicou relação direta e positiva entre comportamentos alimentares disfuncionais e o desejo de ter uma aparência corporal mais magra ${ }^{9}$. Tal fato parece ser relevante, uma vez que em nossa pesquisa muitos participantes afirmaram que se sentem motivados a seguir uma dieta ao observar corpos disseminados como "belos" pela mídia, ainda que sem um acompanhamento nutricional adequado por um nutricionista. Isso se deve, em especial, ao fato de que a mídia promove um padrão de magreza como um estereótipo corporal, ligado a status e ascensão social, competência e atratividade sexual, capaz de perpassar todas as camadas sociais ${ }^{34}$. A mídia social dissemina a fantasia de que, com dedicação e boa vontade, todo e qualquer indivíduo terá a capacidade de adquirir a imagem corporal desejada ou o corpo perfeito, gerando uma pressão pelo emagrecimento a qualquer custo ${ }^{34}$. Dessa forma, o corpo assume o papel de responsável perante as contingências negativas em que o indivíduo está inserido. Há uma validação social diante a regra criada, em que se acredita que o emagrecimento está diretamente ligado ao bem-estar emocional e social. Essa validação a transforma em verdade na sociedade, fazendo com que a magreza como padrão estético dominante seja um fator responsável pelo desenvolvimento de transtornos alimentares ${ }^{34}$.

Quando questionados sobre a utilização de fármacos com o objetivo de emagrecimento, mais da metade dos estudantes participantes do presente estudo relataram a utilização influenciada por algum canal de mídia. Resultados semelhantes foram encontrados em um estudo que analisou a influência dos padrões de beleza veiculados pela mídia como fator decisório na automedicação com moderadores de apetite ${ }^{35}$. No estudo citado anteriormente, foi verificado que $16 \%$ das mulheres utilizam ou já utilizaram moderadores de apetite por influência da internet, 12\% por influência da mídia, 12\% influenciadas por amigos, $6 \%$ por causa da família, $4 \%$ por orientações/causas médicas e $5 \%$ em decorrência de outros fatores ${ }^{35}$.

$\mathrm{Na}$ presente pesquisa, verificou-se ainda, a partir das análises de regressão logística binária, que as variáveis mais importantes na predição dos comportamentos alimentares disfuncionais são: "Já consumi alimentos propostos pela mídia com o intuito de perda de peso" e "Ao observar corpos disseminados como 'belos' pela mídia, me sinto motivado 
a seguir uma dieta sem um acompanhamento nutricional adequado por um nutricionista". Consumir alimentos propostos pela mídia com o objetivo de perda de peso aumentou o risco para desenvolvimento de comportamentos de risco para transtornos alimentares em aproximadamente oito vezes, ao passo que seguir uma dieta sem acompanhamento nutricional por um nutricionista aumentou o risco em aproximadamente três vezes.

A literatura aponta que estudantes de Nutrição com maior insatisfação corporal apresentam maior chance de risco para o desenvolvimento de transtornos alimentares ${ }^{5,15}$. É interessante observar que os dados de nosso estudo apontam que o melhor preditor para os comportamentos alimentares disfuncionais é exatamente o uso da mídia para adoção de comportamentos alimentares com foco na perda de peso e sem acompanhamento nutricional adequado. Esse fato é alarmante, justamente por se tratar de futuros profissionais que terão o comer e o alimento como fonte de estudo, pesquisa e prática profissional.

Diversos fatores influenciam o comportamento alimentar dos sujeitos ${ }^{1,2}$. Em jovens adultos, há estudos que destacam o ingresso na universidade ${ }^{3,4,6}$ e a influência do uso da mídia social $\left.\right|^{3,48-10}$. Devem-se destacar nesse contexto alguns grupos específicos, como o de estudantes de Nutrição, que vem sendo descrito como aquele que apresenta escores elevados de preconceito, quando questionado sobre adiposidade corporal ${ }^{36}$, e elevada frequência de comportamentos alimentares disfuncionais ${ }^{17,19,28}$.

Estudos desse tipo são importantes, pois esses são os futuros profissionais que irão lidar com o público, e suas preocupações com o próprio corpo, seus hábitos e práticas alimentares influenciados pela mídia podem refletir na sua prática profissional e influenciar seus clientes ${ }^{16-18}$. Logo, nossos resultados acrescentam importantes informações que devem ser consideradas ao longo da formação acadêmica dos estudantes de Nutrição. Sendo assim, estratégias devem ser desenvolvidas, de modo que crenças disfuncionais e atitudes alimentares inadequadas não sejam desenvolvidas nessa população.

No campo de prevenção dos transtornos alimentares, algumas estratégias já foram desenvolvidas, com destaque para programas de prevenção direcionados para populações seletivas, ou seja, aquelas como maiores riscos de desenvolvimento de transtornos alimentares ${ }^{37}$, como é o caso de estudantes de Nutrição. Programas preventivos com base na dissonância cognitiva têm demonstrado elevada eficácia e eficiência em populações seletivas ${ }^{11,37}$ e podem ser implementados no contexto de formação de estudantes de Nutrição como uma estratégia de prevenção de desenvolvimento de transtornos alimentares.

Apesar de acrescentar importantes dados à literatura científica sobre a associação do uso da mídia social e os comportamentos alimentares disfuncionais em estudantes de Nutrição, este estudo apresenta algumas limitações que merecem destaque. Podemos citar como limitação o número amostral reduzido e centrado em estudantes de Nutrição de uma única instituição de ensino superior, não permitindo generalizações. Contudo, o cálculo amostral indica que o número de estudantes avaliados é suficiente para as interpretações e a análise conduzidas. Ademais, observamos prevalência de comportamentos alimentares disfuncionais similar àquela demonstrada em estudos prévios ${ }^{17,19,28}$, o que indica que nossa amostra pode ser representativa desse grupo de estudantes de Nutrição. Outra limitação se refere à não separação de homens e mulheres, pois se sabe que atitudes e comportamentos alimentares podem se diferir entre os sexos ${ }^{10,38}$. No entanto, devido à baixa representatividade de homens na presente pesquisa, optou-se por incluí-los na análise conjunta dos dados. Há de se destacar o fato de que é reduzido o número de homens em cursos de Nutrição, majoritariamente frequentados por mulheres.

\section{CONCLUSÕES}

Os resultados apresentados neste estudo demonstram associação entre o uso da mídia social e comportamentos alimentares disfuncionais em estudantes de Nutrição. Entre os fatores preditores dos comportamentos alimentares disfuncionais, foram observadas maiores razões de chances para o desenvolvimento de comportamentos de risco para transtornos alimentares quando há consumo de alimentos para a perda de peso e motivação para seguir dieta sem acompanhamento nutricional.

\section{CONTRIBUIÇÕES INDIVIDUAIS}

Liliane Cupertino de Assis e Pedro Henrique Berbert de Carvalho - Foram responsáveis pela concepção e desenho do estudo, análise e interpretação dos dados, e elaboração do conteúdo.

Camyla Rocha de Carvalho Guedine - Auxiliou na interpretação dos dados e na elaboração do conteúdo.

Todos os autores foram responsáveis pela revisão crítica e aprovação da versão final.

\section{CONFLITO DE INTERESSES}

Os autores não possuem conflito de interesses a serem declarados.

\section{AGRADECIMENTOS}

À coordenação de curso, à chefia de departamento e aos docentes do curso de Nutrição da instituição na qual os dados 
foram coletados. Aos estudantes de Nutrição participantes da presente pesquisa.

\section{REFERÊNCIAS}

1. Quaioti TCB, Almeida SS. Determinantes psicobiológicos do comportamento alimentar: uma ênfase em fatores ambientais que contribuem para a obesidade. Psicol USP. 2006;17(4):193-211.

2. Alvarenga MS, Figueiredo M, Timerman F, Antonaccio CMA. Nutrição Comportamental. 2a ed. Barueri (SP): Manole; 2019.

3. Klassen KM, Douglass CH, Brennan L, Truby H, Lim MSC. Social media use for nutrition outcomes in young adults: a mixed-methods systematic review. Int J Behav Nutr Phys Act. 2018:15(1):1-18.

4. Deliens T, Clarys P, De Bourdeaudhuij I, Deforche B. Determinants of eating behaviour in university students: a qualitative study using focus group discussions. BMC Public Health. 2014;14:1-12.

5. Souza AC, Alvarenga MS. Insatisfação com a imagem corporal em estudantes universitários - Uma revisão integrativa. J Bras Psiquiatr. 2016;65(3):286-99.

6. Barros MML. Trajetórias de jovens adultos: ciclo de vida e mobilidade social. Horizontes Antropol. 2010;16(34):71-92

7. Pereira RF, Alvarenga MS. Disordered eating: identifying, treating, preventing, and differentiating it from eating disorders. Diab Spectr. 2007;20(3):141-8.

8. Sidani JE, Shensa A, Hoffman B, Hanmer J, Primack BA. The association between social media use and eating concerns among US young adults. J Acad Nutr Diet. 2016;116(9):1465-72.

9. Aparicio-Martinez P, Perea-Moreno AJ, Martinez-Jimenez MP, Redel-Macías MD, Pagliari C, Vaquero-Abellan M. Social media, thin-ideal, body dissatisfaction and disordered eating attitudes: An exploratory analysis. Int J Environ Res Public Health. 2019;16(21):1-16.

10. Rodgers RF, Slater A, Gordon CS, McLean SA, Jarman HK, Paxton SJ. A Biopsychosocial model of social media use and body image concerns, disordered eating, and musclebuilding behaviors among adolescent girls and boys. J Youth Adolesc. 2020;49:399-409.

11. Amaral ACS, Stice E, Ferreira MEC. A controlled trial of a dissonance-based eating disorders prevention program with Brazilian girls. Psicol Reflex Crít. 2019;32(1):e13.

12. Mingoia J, Hutchinson AD, Gleaves DH, Wilson C. The relationship between posting and photo manipulation activities on social networking sites and internalization of a tanned ideal among Australian adolescents and young adults. Soc Media Soc. 2019;1(1):1-10.

13. Karazsia BT, van Dulmen MH, Wong K, Crowther JH. Thinking meta-theoretically about the role of internalization in the development of body dissatisfaction and body change behaviors. Body Image. 2013;10(4):433-41.

14. Chassiakos YLR, Radesky J, Christakis D, Moreno MA, Cross C. Children and adolescents and digital media. Pediatrics. 2016;138(5):e20162593.

15. Poínhos R, Alves D, Vieira E, Pinhão S, Oliveira BM, Correia F. Eating behaviour among undergraduate students. Comparing nutrition students with other courses. Appetite. 2015;84:28-33.

16. Bosi MLM, Luiz RR, Morgado CMC, Costa MLS, Carvalho RJ. Autopercepção da imagem corporal entre estudantes de nutrição: um estudo no município do Rio de Janeiro. J Bras Psiquiatr. 2006;55(2):108-13.

17. Mahn HM, Lordly D. A review of eating disorders and disordered eating amongst nutrition students and dietetic professionals. Can J Diet Pract Res. 2015;76(1):38-43.

18. Bandeira YER, Mendes ALRF, Cavalcante ACM, Arruda SPM. Avaliação da imagem corporal de estudantes do curso de Nutrição de um centro universitário particular de Fortaleza. J Bras Psiquiatr. 2016;65(2):168-73.
19. Alvarenga MS, Scagliusi FB, Philippi ST. Comportamento de risco para transtorno alimentar em universitárias brasileiras. Rev Psiquiatr Clin. 2011;38(1):3-7.

20. Korinth A, Schiess S, Westenhoefer J. Eating behaviour and eating disorders in students of nutrition sciences. Public Health Nutr. 2010;13(1):32-7.

21. Oliveira PL, Ferreira MEC, Neves CM, Meireles JFF, Carvalho PHB. Insatisfação, checagem corporal e comportamentos de risco para transtornos alimentares em estudantes de cursos da saúde. J Bras Psiquiatr. 2017;66(4):216-20.

22. Silva NLN, Soares TO, Neves CM, Meireles JFF, Carvalho PHB, Ferreira MEC. Insatisfação e checagem corporal e comportamento alimentar em estudantes de Educação Física, Nutrição e Estética. Rev Bras Ciênc Mov. 2017;25(2):99-106.

23. Moraes JMM, Oliveira AC, Nunes PP, de Lima MTMA, de Oliveira Abreu JA, Arruda SPM. Fatores associados à insatisfação corporal e comportamentos de risco para transtornos alimentares entre estudantes de Nutrição. Rev Pesq Saúde. 2016;17(2):106-11.

24. Smith AR, Hames JL, Joiner Jr TE. Status update: Maladaptive Facebook usage predicts increases in body dissatisfaction and bulimic symptoms. J Affect Disord. 2013;149(13):235-40.

25. Holland G, Tiggemann M. A systematic review of the impact of the use of social networking sites on body image and disordered eating outcomes. Body Image. 2016;17:100-10.

26. Mabe AG, Forney KJ, Keel PK. Do you "like" my photo? Facebook use maintains eating disorder risk. Int J Eat Disord. 2014;47(5):516-23.

27. Wilksch SM, O'Shea A, Ho P, Byrne S, Wade TD. The relationship between social media use and disordered eating in young adolescents. Int J Eat Disord. 2019;56(1):96-106.

28. Eisenberg D, Nicklett EJ, Roeder K, Kirz NE. Eating disorder symptoms among college students: prevalence, persistence, correlates, and treatment-seeking. J Am Coll Health. 2011;59(8):700-7.

29. World Health Organization. Physical status: The use of and interpretation of anthropometry. Report of a WHO Expert Committee: Geneva, Switzerland: World Health Organization; 1995.

30. Associação Brasileira de Empresas de Pesquisa (ABEP). Critério de Classificação Econômica Brasil. São Paulo: ABEP; 2018

31. Nunes MA, Camey S, Olinto MTA, Mari JJ. The validity and 4-year test-retest reliability of the Brazilian version of the Eating Attitudes Test-26. Braz J Med Biol Res. 2005;38(11):1655-62.

32. Garner DM, Olmsted MP, Bohr Y, Garfinkel PE. The eating attitudes test: psychometric features and clinical correlates. Psychol Med. 1982;12(4):871-8.

33. Trettoa MB, Laina N, Pereira FB. Dietas da moda versus distúrbios alimentares. V Congresso de Pesquisa e Extensão da FSG; Centro Universitário da Serra Gaúcha; 2017. p. 110-2.

34. do Vale AM0, Elias LR. Transtornos alimentares: uma perspectiva analíticocomportamental. Rev Bras Terap Comport Cog. 2011;13(1):52-70.

35. Dutra JR, da Fonseca Souza SM, Peixoto MC. A influência dos padrões de beleza veiculados pela mídia, como fator decisório na automedicação com moderadores de apetite por mulheres no município de Miracema-RJ. Rev Transformar. 2015;7:194-213.

36. Obara AA, Vivolo SRGF, Alvarenga MS. Preconceito relacionado ao peso na conduta nutricional: um estudo com estudantes de nutrição. Cad Saúde Pública. 2018;34(8):e00088017.

37. Stice E, Mart, CN, Shaw H, Rohde P. Meta-analytic review of dissonance-based eating disorder prevention programs: Intervention, participant, and facilitator features that predict larger effects. Clin Psychol Rev. 2019;70:91-107.

38. Murray SB, Nagata JM, Griffiths S, Calzo JP, Brown TA, Mitchison D, et al. The enigma of male eating disorders: a critical review and synthesis. Clin Psychol Rev. 2017;57:1-11. 